\title{
Anti-periodic Boundary Value Problem for Impulsive Differ- ential Equations with Delay
}

FEnGQIN ZHANG

Department of Mathematics, Yuncheng University, Yuncheng Shanxi 044000, China e-mail : zhafq@263.net

Abstract. The method of upper and lower solutions coupled with monotone iterative technique is used to obtain the results of existence and uniqueness for an anti-periodic boundary value problem of impulsive differential equations with delay.

\section{Introduction}

We are concerned with the following anti-periodic boundary value problem (APBVP for short) for a first-order impulsive differential equation with delay in $R$

$$
\left\{\begin{array}{l}
x^{\prime}(t)=f\left(t, x(t), x_{t}\right), \quad t \in J^{\prime} \\
\Delta x\left(t_{k}\right)=I_{k}\left(x\left(t_{k}\right)\right), \quad k=1,2, \cdots, m \\
x(\theta) \equiv x(0)=-x(T), \quad \theta \in[-\tau, 0]
\end{array}\right.
$$

where $f: J \times R \times D \rightarrow R, D=L^{1}([-r, 0], R), I_{k} \in C(R, R), \Delta x\left(t_{k}\right)$ represents the jump of $x(t)$ at $t=t_{k}$, i.e., $\Delta x\left(t_{k}\right)=x\left(t_{k}^{+}\right)-x\left(t_{k}^{-}\right)$for all $k=1,2, \cdots, m, 0<t_{1}<$ $t_{2}<\cdots<t_{m}<T, \delta=\max \left\{t_{k}-t_{k-1} ; k=1,2, \cdots, m+1\right\}$ here $t_{0}=0, t_{m+1}=T$; $\tau>0, J=[0, T], J^{\prime}=J \backslash\left\{t_{1}, t_{2}, \cdots, t_{m}\right\}$; for every $t \in J, x_{t} \in D$ is defined by $x_{t}(s)=x(t+s),-\tau \leq s \leq 0$.

Suppose $J_{0}=[-\tau, T]$. Let $P C\left(J_{0}, R\right)=\left\{x: J_{0} \rightarrow R, x(t)\right.$ is continuous for $t \in J_{0}, t \neq t_{k}$ and $x\left(t_{k}^{+}\right), x\left(t_{k}^{-}\right)$exist and $x\left(t_{k}\right)=x\left(t_{k}^{-}\right)$for $k=$ $1,2, \cdots, m\} ; P C^{\prime}(J, R)=\{x: J \rightarrow R, x(t)$ is continuously differentiable for $t \in J^{\prime}$ and $x\left(t_{k}^{+}\right), x\left(t_{k}^{-}\right)$exist and $x^{\prime}\left(t_{k}\right)=x^{\prime}\left(t_{k}^{-}\right)$for $\left.k=1,2, \cdots, m\right\} ; E=$ $P C\left(J_{0}, R\right) \bigcap P C^{\prime}(J, R)$. Obviously, for any $t \in J$ and $x \in E$, we have $x_{t} \in D$ and $P C\left(J_{0}, R\right)$ and $E$ are Banach spaces with the norms,

$$
\|x\|_{P C\left(J_{0}, R\right)}=\sup \left\{|x(t)|: t \in J_{0}\right\},\|x\|_{E}=\|x\|_{P C(J, R)}+\left\|x^{\prime}\right\|_{P C(J, R)},
$$

where $\left\|x^{\prime}\right\|_{P C(J, R)}=\sup \left\{\left|x^{\prime}(t)\right|: t \in J\right\}$. By a solution of (1.1) mean $x \in E$ for which problem (1.1) is satisfied.

Received August 29, 2005, and, in revised form, March 6, 2007.

2000 Mathematics Subject Classification: 34B15, 34B37, 34K10.

Key words and phrases: anti-periodic boundary value problem, monotone iterative technique, lower and upper related solutions.

This work is supported by the Sciences Foundation of Shanxi(20051010) and the Major Subject Foundation of Shanxi. 
Impulsive differential equations with delay have been extensively studied; see [1]-[4]. Those results are applicable in some important cases such as the initial or the periodic case. However they are not valid, for example, for anti-periodic $x(0)+x(T)=0$. The APBVPs have been studied by many authors; see [5]-[7] and references therein.

It is the purpose of the present paper to establish the existence and uniqueness of solution for (1.1) .

\section{Comparison theorems}

This section is devoted to comparison theorems, which are needed for the successful employment of the monotone iterative technique.

Lemma 2.1([7]). Let $p \in E$ such that

$$
\left\{\begin{array}{l}
p^{\prime}(t) \leq-M p(t)-N \min _{s \in[-\tau, 0]} p_{t}(s), \quad t \in J^{\prime}, \\
p\left(t_{k}^{+}\right) \leq\left(1-L_{k}\right) p\left(t_{k}\right), \quad k=1,2, \cdots, m, \\
p(0) \leq p(\theta) \leq 0, \quad \theta \in[-\tau, 0],
\end{array}\right.
$$

where $M, N>0,0 \leq L_{k}<1(k=1,2, \cdots, m)$ are constants such that for any positive integer $n_{1}, n_{2}: 1 \leq n_{1} \leq n_{2} \leq m$

$$
\prod_{k=n_{1}}^{n_{2}}\left(1-L_{k}\right) \geq N e^{M \tau} \delta\left[1+\sum_{k=n_{1}}^{n_{2}} \prod_{j=k}^{n_{2}}\left(1-L_{j}\right)\right] .
$$

Then $p(t) \leq 0$ on $J_{0}$.

For any $p(t) \in E$, we have

$$
\min _{s \in[-\tau, 0]}\left(-p_{t}(s)\right) \leq-p_{t}(s) \leq-\min _{s \in[-\tau, 0]} p_{t}(s) .
$$

Therefore, we get the following corollary.

Corollary 2.1. If $p \in E$ such that

$$
\left\{\begin{array}{l}
p^{\prime}(t) \geq-M p(t)-N \min _{s \in[-\tau, 0]} p_{t}(s), \quad t \in J^{\prime}, \\
p\left(t_{k}^{+}\right) \geq\left(1-L_{k}\right) p\left(t_{k}\right), \quad k=1,2, \cdots, m, \\
p(0)=p(\theta) \geq 0, \quad \theta \in[-\tau, 0] .
\end{array}\right.
$$

And other conditions of Lemma 2.1 hold. Then $p(t) \geq 0$ on $J_{0}$.

Lemma 2.2([1]). Let $p(t) \in E$ such that

$$
\left\{\begin{array}{l}
p^{\prime}(t) \leq-M p(t)+N \min _{s \in[-\tau, 0]} p_{t}(s), \quad t \in J^{\prime}, \\
p\left(t_{k}^{+}\right) \leq\left(1-L_{k}\right) p\left(t_{k}\right), \quad k=1,2, \cdots, m, \\
p(0)=p(\theta) \leq p(T), \quad t \in[-\tau, 0],
\end{array}\right.
$$


where $M>0, N \geq 0,0 \leq L_{k}<1, k=1,2, \cdots, m, \tau>0$ are constants such that for any positive integer: $1 \leq n_{1} \leq n_{2} \leq m$,

$$
c=\frac{N\left[\sum_{i=0}^{m-1} \prod_{t_{i}<t_{k}<T}\left(1-L_{i}\right) \exp \left[-M\left(T-t_{i+1}\right)\right]\right]}{M\left[1-\prod_{k=1}^{m}\left(1-L_{k}\right) \exp (-M T)\right]}<1, \quad \bar{t} \in\left(t_{1}, T\right] .
$$

Then $p(t) \leq 0$ on $J_{0}$.

Lemma 2.3([8]). Let $F$ be a Banach space and $\hat{E}=C([a, b], F)$. Let $S: \hat{E} \rightarrow F$ be an operator for which

$$
\|S \varphi-S \psi\|_{F} \leq \epsilon\|\varphi-\psi\|_{\hat{E}}, 0 \leq \epsilon<1 .
$$

Then for any point $\xi \in[a, b]$ there exist an element $\phi \in \hat{E}$ such that $S \phi=\phi(\xi)$.

\section{Results}

In this section, we first consider the linear APBVPs

$$
\left\{\begin{array}{l}
p^{\prime}(t)+M p(t)+N p_{t}(s)=\sigma(t), \quad t \in J^{\prime} \\
\Delta p\left(t_{k}\right)=L_{k} p\left(t_{k}\right)+\sigma_{1}\left(t_{k}\right), \quad k=1,2, \cdots, m \\
p(0) \equiv p(\theta)), \quad \theta \in[-\tau, 0] \\
p(0)+p(T)=0
\end{array}\right.
$$

where $\sigma: R \rightarrow R$ continuous. From Lemma 2.1 and Corollary 2.1 in Section 2, we can get the following corollaries.

Corollary 3.1. Assume that (2.2) hold. Then (3.1) has at most one solution.

Theorem 3.1. Assume that $M, N, L_{k}(k=1,2, \cdots, m)$ satisfy the condition (2.2) and (2.3). Then (3.1) possesses a unique solution.

Proof. For any $t \in J$, we have

$$
\begin{aligned}
p(t)= & p(0) \prod_{0<t_{k}<t}\left(1-L_{k}\right) \exp (-M t) \\
& +\int_{0}^{t} \prod_{0<t_{k}<t}\left(1-L_{k}\right) \exp [-M(t-s)]\left(\sigma(s)-N p_{t}(s)\right) d s \\
& +\sum_{0<t_{k}<t} \prod_{t_{k}<t_{i}<t}\left(1-L_{i}\right) \exp \left[-M\left(t-t_{k}\right)\right] \sigma_{1}\left(t_{k}\right) .
\end{aligned}
$$

Let $t=T$, we obtain

$$
\begin{aligned}
p(0)= & {\left[1+\prod_{k=1}^{m}\left(1-L_{k}\right) \exp (-M T)\right]^{-1} } \\
& \left\{-\int_{0}^{T} \prod_{0<t_{k}<T}\left(1-L_{k}\right) \exp [-M(T-s)]\left(\sigma(s)-N p_{t}(s)\right) d s\right. \\
& \left.-\sum_{0<t_{k}<T} \prod_{t_{k}<t_{i}<T}\left(1-L_{i}\right) \exp \left[-M\left(T-t_{k}\right)\right] \sigma_{1}\left(t_{k}\right)\right\} .
\end{aligned}
$$


Define the operator $B: E_{0} \rightarrow R$ by the equality

$$
\begin{aligned}
B p= & {\left[1+\prod_{k=1}^{m}\left(1-L_{k}\right) \exp (-M T)\right]^{-1} } \\
& \left\{-\int_{0}^{T} \prod_{0<t_{k}<T}\left(1-L_{k}\right) \exp [-M(T-s)]\left(\sigma(s)-N p_{t}(s)\right) d s\right. \\
& \left.-\sum_{0<t_{k}<T} \prod_{t_{k}<t_{i}<T}\left(1-L_{i}\right) \exp \left[-M\left(T-t_{k}\right)\right] \sigma_{1}\left(t_{k}\right)\right\},
\end{aligned}
$$

where $E_{0}=\left\{x \in P C\left(J_{0}, R\right): x(\theta) \equiv x(0), \theta \in[-\tau, 0]\right\}$. For any $p, q \in E_{0}$ we have

$$
\begin{aligned}
& |B p-B q| \leq\left[1+\prod_{k=1}^{m}\left(1-L_{k}\right) \exp (-M T)\right]^{-1} N|p-q|_{0} \\
& \times \int_{0}^{T} \prod_{0<t_{k}<T}\left(1-L_{k}\right) \exp [-M(T-s)] d s \leq c|p-q|_{0},
\end{aligned}
$$

i.e., $|B p-B q| \leq c|p-q|_{0}$, where $0<c<1$. By Lemma 2.3, there exists an element $p \in E_{0}$ such that $B p \equiv p(0)$. This implies that there exists a solution $p$ for (3.1). The uniqueness of solutions of (3.1) follows from Corollary 3.1. The proof of the theorem is complete.

Now we give the definition of a pair of lower and upper related solutions.

Definition 3.1. We say $v, w \in E$ are a pair of lower and upper related solutions for (1.1) if they satisfy

$\left\{\begin{array}{l}v^{\prime}(t) \leq f\left(t, v(t), v_{t}\right), \quad t \in J^{\prime} \\ \Delta v\left(t_{k}\right) \leq I_{k}\left(v\left(t_{k}\right)\right), \quad k=1,2, \cdots, m, \\ v(\theta) \equiv v(0) \leq-w(T), \quad \theta \in[-\tau, 0],\end{array} \quad\left\{\begin{array}{l}w^{\prime}(t) \geq f\left(t, w(t), w_{t}\right), \quad t \in J^{\prime}, \\ \Delta w\left(t_{k}\right) \geq I_{k}\left(w\left(t_{k}\right)\right), \quad k=1,2, \cdots, m, \\ w(\theta) \equiv w(0) \geq-v(T), \quad \theta \in[-\tau, 0] .\end{array}\right.\right.$

Let $v, w \in E$ be a pair of lower and upper related solutions of (1.1) such that

$$
v \leq w \quad \text { on } \quad J,
$$

we define the sector $[v, w]=\{u \in E, v(t) \leq u(t) \leq w(t), t \in J\}$.

Let us list the following assumptions for convenience.

$\left(H_{0}\right) \quad f \in C(J \times R \times D, R)$ and $I_{k} \in C(R, R)(k=1,2, \cdots, m)$;

$\left(H_{1}\right) \quad v, w \in E$ are lower and upper related solutions of (1.1) satisfying (3.2);

$\left(H_{2}\right)$ There exist $M, N>0$ such that

$$
\begin{aligned}
& -M(\bar{u}-u)+N\left(\max _{s \in[-\tau, 0]} \bar{\varphi}(s)-\max _{s \in[-\tau, 0]} \varphi(s)\right) \geq f(t, \bar{u}, \bar{\varphi})-f(t, u, \varphi) \\
& \geq-M(\bar{u}-u)-N\left(\max _{s \in[-\tau, 0]} \bar{\varphi}(s)-\max _{s \in[-\tau, 0]} \varphi(s)\right)
\end{aligned}
$$

whenever $v(t) \leq u \leq \bar{u} \leq w(t), v_{t}(s) \leq \varphi(s) \leq \bar{\varphi}(s) \leq w_{t}(s)$ for $t \in J$ and $s \in[-\tau, 0]$, where $u, \bar{u} \in R$ and $\varphi, \bar{\varphi} \in D$;

$\left(H_{3}\right)$ There exist $L_{k}: 0 \leq L_{k}<1(k=1,2, \cdots, m)$ such that $I_{k}(\bar{u})-I_{k}(u) \geq$ $-L_{k}(\bar{u}-u)$ whenever $v\left(t_{k}\right) \leq u \leq \bar{u} \leq w\left(t_{k}\right)$ where $u, \bar{u} \in R$;

$\left(H_{4}\right) \quad M, N, L_{k}(k=1,2, \cdots, m)$ satisfy $(2.2)$ and $(2.3)$.

The following theorem is the most important result that we get.

Theorem 3.2. Suppose that $\left(H_{0}\right)-\left(H_{4}\right)$ hold. Then (1.1) has a unique solution 
$x \in[v, w]$.

Proof. We construct the sequences $\left\{v_{n}\right\},\left\{w_{n}\right\} \subset E$ by defining $v_{1}=v, w_{1}=w$, and for $n>1, v_{n}$ and $w_{n}$ are the solutions of

$$
\left\{\begin{array}{l}
v_{n}^{\prime}(t)=f\left(t, v_{n-1}(t), v_{n-1_{t}}\right)-M\left[v_{n}(t)-v_{n-1}(t)\right] \\
-N\left[\max _{s \in[-\tau, 0]} v_{n_{t}}(s)-\max _{s \in[-\tau, 0]} v_{n-1_{t}}(s)\right] \\
\Delta v_{n}\left(t_{k}\right)=-L_{k} v_{n}\left(t_{k}\right)+I_{k}\left(v_{n-1}\left(t_{k}\right)\right)+L_{k} v_{n-1}\left(t_{k}\right), k=1,2, \cdots, m \\
v_{n}(0)=-w_{n-1}(T) \\
v_{n}(\theta) \equiv v_{n}(0), \theta \in[-\tau, 0]
\end{array}\right.
$$

and

$$
\left\{\begin{array}{l}
w_{n}^{\prime}(t)=f\left(t, w_{n-1}(t), w_{n-1_{t}}\right)-M\left[w_{n}(t)-w_{n-1}(t)\right] \\
-N\left[\max _{s \in[-\tau, 0]} w_{n_{t}}(s)-\max _{s \in[-\tau, 0]} w_{n-1_{t}}(s)\right] \\
\Delta w_{n}\left(t_{k}\right)=-L_{k} w_{n}\left(t_{k}\right)+I_{k}\left(w_{n-1}\left(t_{k}\right)\right)+L_{k} w_{n-1}\left(t_{k}\right), k=1,2, \cdots, m \\
w_{n}(0)=-v_{n-1}(T) \\
w_{n}(\theta) \equiv w_{n}(0), \theta \in[-\tau, 0] .
\end{array}\right.
$$

Obviously, the existence and uniqueness of solutions for (3.3) and (3.4) are guaranteed.

We prove that $v_{1} \leq v_{2} \leq w_{2} \leq w_{1}$. In fact, if we consider $\alpha=v_{1}-v_{2}$, by $\left(H_{2}\right)$ and Lemma 2.1 we have $\alpha(t)=v_{1}-v_{2} \leq 0$ on $J_{0}$. Analogously, $w_{2} \leq w_{1}$ on $J_{0}$. Now, let $\alpha_{1}=v_{2}-w_{2}$, using $\left(H_{2}\right)$ and Lemma 2.1, $\alpha_{1}=v_{2}-w_{2} \leq 0$ on $J$. It is therefore easy to see that these sequences satisfy the property $v_{n} \leq v_{n+1} \leq w_{n+1} \leq$ $w_{n}, \quad n \geq 1$.

We have two monotone sequences that are bounded. By standard arguments[9], there exist $\rho$ and $\mu$ with $\left\{v_{n}\right\} \nearrow \rho$ and $\left\{w_{n}\right\} \searrow \mu$ and $\rho \leq \mu$. Moreover the convergence is uniform on $J$. Also, we obtain that the function $\rho, \mu$ satisfy $\left\{\begin{array}{l}\rho^{\prime}(t)=f\left(t, \rho(t), \rho_{t}\right), t \in J^{\prime} \\ \Delta \rho\left(t_{k}\right)=I_{k}\left(\rho\left(t_{k}\right)\right), k=1,2, \cdots, m, \\ \rho(\theta) \equiv \rho(0), \theta \in[-\tau, 0] \\ \rho(0)+\mu(T)=0,\end{array} \quad\left\{\begin{array}{l}\mu^{\prime}(t)=f\left(t, \mu(t), \mu_{t}\right), t \in J^{\prime} \\ \Delta \mu\left(t_{k}\right)=I_{k}\left(\rho\left(t_{k}\right)\right), k=1,2, \cdots, m, \\ \mu(\theta) \equiv \mu(0), \theta \in[-\tau, 0] \\ \mu(0)+\rho(T))=0 .\end{array}\right.\right.$

If we show that $\rho=\mu$, then $\rho$ is a solution of (1.1). Consider $p=\rho-\mu$. By Lemma 2.2, we get $p(t)=\rho(t)-\mu(t) \geq 0$ for $t \in J$. Hence we have $\rho \equiv \mu$ for $t \in J$.

If $x(t) \in[v, w]$ is a solution of (1.1), one can see that $v_{n} \leq x \leq w_{n}, n=1,2, \cdots$. In fact, we suppose $v_{n_{0}} \leq x \leq w_{n_{0}}$. Let $\alpha_{2}(t)=v_{n_{0}+1}(t)-x(t)$, then we can get that

$$
\left\{\begin{array}{l}
\alpha_{2}^{\prime}(t) \leq-M \alpha_{2}(t)-N \min _{s \in[-\tau, 0]} \alpha_{2_{t}}(s) \\
\Delta \alpha_{2}\left(t_{k}\right) \leq-L_{k} \alpha_{2}\left(t_{k}\right), k=1,2, \cdots, m \\
\alpha_{2}(0)=-w_{n_{0}}(T)+x(T) \leq 0 \\
\alpha_{2}(\theta) \equiv \alpha_{2}(0), \theta \in[-\tau, 0]
\end{array}\right.
$$

By Lemma 2.1, we have $\alpha_{2}(t) \leq 0$ on $J$, that is, $v_{n_{0}+1}(t) \leq x(t)$. Analogously, $x(t) \leq w_{n_{0}+1}(t)$ on $J$. Therefore, $v_{n} \leq x \leq w_{n}(n=1,2, \cdots)$ by induction. Thus, 
passing to the limit, we may conclude that $\rho \leq x \leq \mu$, that is, $\rho \equiv \mu \equiv x$. Therefore, The proof of the theorem is completed.

Example. Consider the anti-periodic boundary value problem of the impulsive equation

$$
\left\{\begin{array}{l}
x^{\prime}(t)=-x-\frac{3}{2 e} \sin x_{t}+\left(1+\frac{3}{2 e}\right)(1-t), \quad t \in[0,1], t \neq \frac{1}{2}, \\
\Delta x\left(\frac{1}{2}\right)=-\frac{1}{2} x\left(\frac{1}{2}\right), \\
x(\theta) \equiv x(0)=-x(1), \quad \theta \in\left[-\frac{1}{2}, 0\right],
\end{array}\right.
$$

where $x_{t}=x(t+s), s \in\left[-\frac{1}{2}, 0\right]$.

$$
\text { Let } v(t)=\left\{\begin{array}{cc}
-1, & 0 \leq t \leq \frac{1}{2}, \\
-\frac{1}{2}, & \frac{1}{2}<t \leq 1,
\end{array} \quad w(t)=\left\{\begin{array}{cc}
1, & 0 \leq t \leq \frac{1}{2}, \\
\frac{1}{2}, & \frac{1}{2}<t \leq 1 .
\end{array} \quad \text { Then } v(t), w(t)\right.\right.
$$

are a pair of lower and upper related solutions for (3.5). And (3.5) satisfies all the conditions of Theorem 3.2, therefore, we have a unique solution of (3.5) by Theorem 3.2 .

\section{References}

[1] Z. He and W. Ge, Periodic boundary value for first order impulsive delay differential equations, Applied Math. Comput., 104(1999), 51-63.

[2] G. Ballinger and X. Liu, Existence, uniqueness and boundedness results for impulsive delay differential equations, Appl. Anal., 74(2000), 71-93.

[3] F. Zhang, M. Li and J. Yan, Non-homogeneous Boundary Value Problem for First Order Impulsive Differential Equations with Delay, Comput. Appl. Math., 51(2006), 927-936.

[4] M. Li, M. Wang and F. Zhang, Controllability of Impulsive Functional differential Systems in Banach Spaces, Chaos,Solitons \& Fractals, 29(2006), 175-181.

[5] Y. Yin, Monotone iterative technique and quasilinearization for some anti -periodic problems, Nonlinear Word, 3(1996), 253-262.

[6] D. Fraco and J. J. Nieto, First-order impulsive ordinary differential equations with anti-periodic and nonlinear boundary conditions, Nonlinear Anal, 42(2000), 163-173.

[7] F. Zhang and Z. Ma, Anti-periodic and Nonlinear Boundary Problems for First Order Differential Equations with Piecewise Constant Arguments, Acta Math. Scientia, 23A(6)(2003), 641-649. (Chinese)

[8] S. R. Bernfeld, V.Lakshmikantham and Y. M. Reddy, Fixed point theorems systems, Appl. Anal., 6(1977), 271-280.

[9] G. S. Ladde, V. Lakshmikantham and A. S. Vatsala, Monotone iterative techniques for nonlinear differential equations, Pitman Boston, 1985. 